\title{
Lemierre syndrome in a child with recent pharyngitis
}

\author{
Jonnathan Moore Busko, MD, MPH; ${ }^{*}$ Wayne Triner, DO, $\mathrm{MPH}^{\dagger}$
}

\begin{abstract}
A 14-year-old boy presented with fever and progressive respiratory distress, one week after an episode of pharyngitis. Although there was a concern about pulmonary embolism secondary to a lower extremity fracture, his presentation was most consistent with Lemierre syndrome. This syndrome is an uncommon but potentially lethal complication of otolaryngological infections. Early recognition and aggressive antibiotic therapy are critical elements in reducing mortality. Emergency physicians should be aware of this syndrome because its incidence appears to be increasing.
\end{abstract}

Key words: pediatric; infectious disease; fusobacter; Lemierre syndrome; diagnosis, treatment

\section{RÉSUMÉ}

Un adolescent âgé de 14 ans s'est présenté à l'urgence avec de la fièvre et une détresse respiratoire progressive, une semaine après un épisode de pharyngite. Bien qu'une embolie pulmonaire découlant d'une fracture à une extrémité inférieure ait été évoquée, les symptômes de présentation étaient plus évocateurs du syndrome de Lemierre. Ce syndrome est une complication peu courante mais potentiellement fatale des infections oto-laryngologiques. Son identification précoce et une antibiothérapie agressive sont des éléments cruciaux dans la réduction de la mortalité. Les médecins d'urgence doivent être sensibilisés à ce syndrome, car son incidence semble être en hausse.

\section{Introduction}

In the pre-antibiotic era, disease processes that today are effectively managed with antibiotics were often associated with fatal complications. In 1936, Lemierre described a syndrome referred to as postanginal septicemia ${ }^{1}$ or necrobacillosis, ${ }^{2}$ which consists of acute oropharyngeal infection, septic thrombophlebitis of the internal jugular vein, septicemia and metastatic septic emboli. We report the case of a 14-year-old boy who presented to a large aca- demic emergency department (ED) with fever and septic pulmonary emboli related to Lemierre syndrome.

\section{Case report}

A 14-year-old boy was referred to the ED by his primary physician for evaluation of a fever and chest nodules on $\mathrm{x}$ ray. Approximately 6 weeks prior to presentation, he had suffered left tibial and fibular fractures requiring closed reduction under general anesthesia. His recovery was un-

\footnotetext{
*Carolinas Medical Center, Department of Emergency Medicine, 1000 Blythe Blvd., Charlotte, NC

tAlbany Medical Center, Department of Emergency Medicine, MC-139, Albany, NY
}

Received: Oct. 29, 2003; final submission: Mar. 30, 2004; accepted: Apr. 5, 2004

This article has been peer reviewed.

Can J Emerg Med 2004;6(4):285-7 
eventful until 1 week before presentation, when he developed an exudative pharyngitis, for which rapid streptococcal, throat culture, and Mono-spot tests were negative. This pharyngitis improved without treatment over approximately 2 days.

After the pharyngitis but 3 days prior to his ED presentation, the patient developed dyspnea, tachypnea and spiking fevers. A chest x-ray demonstrated multiple nodular lesions. He was started on azithromycin and his condition improved over 24 hours.

On the day of ED presentation, he developed suddenly worsening dyspnea, spiking fevers, chills and pleuritic chest pain. A repeat chest X-ray showed an increase in the size and number of lesions. At this point, he was referred to the ED. In the ED, he denied swelling or pain in his fractured extremity. He had had no recent dental work, appliance or central venous line placement, nor any history of valvular heart disease. His social and family histories were non-contributory.

On examination, his vitals signs included a pulse rate of 118 beats $/ \mathrm{min}$, a temperature of $38.6^{\circ} \mathrm{C}$, blood pressure $118 / 76 \mathrm{~mm} \mathrm{Hg}$, respiratory rate 16 breaths/min and oxygen saturation $99 \%$ on room air. Physical exam was remarkable only for pharyngeal erythema with uvular exudates, right-sided neck tenderness with pain on lateral movement and clear and equal but diminished lung sounds. He had mobile right-sided anterior cervical and submandibular lymph nodes. His left tibia and fibula were in a cast and there was no swelling, erythema or rash noted.

The white blood cell count was 11.5 with $14 \%$ bands and $73 \%$ neutrophils. Contrast-enhanced spiral CT of the neck was negative for internal jugular vein thrombosis or abscess, but chest CT showed multiple emboli throughout both lungs. There was no evidence of pneumonia.

The patient was seen by the infectious disease service. He was started on vancomycin, gentamycin and clindamycin. Despite this, his temperature rose to $40^{\circ} \mathrm{C}$ and his oxygen saturations fell to $95 \%$.

A duplex ultrasound of the left lower extremity showed no evidence of deep vein thrombosis (DVT), but an ultrasound of the neck obtained 3 days after admission demonstrated right internal jugular thrombosis. The patient remained on IV antibiotics and continued to improve. After 4 days, his blood cultures grew Fusobacterium necrophorum, and his antibiotic spectrum was narrowed. A peripherally inserted central catheter (PICC) was placed, and he was discharged home on IV antibiotics. He continued to do well and has completely recovered from his infection.

\section{Discussion}

Patients with Lemierre syndrome commonly present with sepsis after apparent resolution of an acute pharyngitis. ${ }^{3}$ Although pharyngitis (often viral) is the most common antecedent otoloaryngological infection, ${ }^{4}$ other infections such as peritonsillar abscesses and otitis media have also been implicated. The key factor in the progression of the disease from an otolaryngologic infection to systemic septic emboli is the invasion of deep neck tissues by anaerobic oral pathogens. ${ }^{1,3,5,6} \mathrm{~A}$ resultant septic thrombophlebitis of the internal jugular vein acts as a seeding point for septic thromboemboli.

The most common sites of metastasis are the lungs; ${ }^{1,3,4,6}$ however, meningitis, osteomyelitis, septic arthritis of the ankle, hips and shoulders, as well as abscesses of the spleen, paravertebral muscles, thigh and skin have been reported, suggesting aggressive systemic dissemination. ${ }^{2}$ Rarely, this syndrome is associated with headache, ${ }^{5}$ carotid thrombosis, ${ }^{6}$ and mediastinitis secondary to extension along the carotid sheath.

Lemierre syndrome is diagnosed by the finding of a characteristic otolaryngologic infection (or a history of the same), bacteremia confirmed by at least 1 positive blood culture, clinical or radiographic evidence of internal jugular thrombophlebitis, and demonstrated septic emboli. ${ }^{2}$ The characteristic pathogen is F. necrophorum, ${ }^{*}$ an oropharyngeal anaerobe. With enriched anaerobic media, cultures are typically positive in 1-3 days. ${ }^{8}$ In the largest published series to date, Sinave and colleagues ${ }^{2}$ identified 36 patients who met strict diagnositic criteria for Lemierre syndrome and found $22(61 \%)$ with definite F. necrophorum bactermia, $8(22 \%)$ with probable $F$. necrophorum bactermia, and $6(17 \%)$ with other organisms, including Streptococcus viridans, Bacteriodes clostridiiformis, Peptostreptococcus magnus, Bacteroides sp, Peptostreptococcus, F. naviforme and Eikenella corrodens. It is possible that these cases reflect difficulty growing this strict anaerobe; it is also possible that multiple pathogens may be responsible for Lemierre syndrome. In fact, one-third of patients with Lemierre syndrome have a polymicrobial bactermia. ${ }^{9}$

Without early detection and treatment, morbidity and mortality are high. Although MRI has been used, recent literature suggests that colour Doppler ultrasonography and high-resolution CT, respectively, play the most important roles in identifying jugular venous thrombosis and pulmonary metastases. ${ }^{10}$ Early in the disease process, CT studies of the neck may be falsely negative, ${ }^{11}$ and in the case

*"Rod shaped organism that brings death" 
described, the initial CT on the day of admission did not demonstrate internal jugular involvement, although a carotid ultrasound done 3 days later did. Serial imaging studies may be necessary, and ultrasound is now recommended as the initial modality to evaluate for internal jugular thrombosis. ${ }^{12,13}$

ED diagnosis and treatment is traditionally limited to a high clinical index of suspicion, early empiric antibiotic administration and subsequent imaging; however, emergency physicians skilled in ultrasound evaluation may be able to improve diagnostic performance and rapidly confirm Lemierre syndrome by identifying internal jugular thrombosis on rapid ED scans. ${ }^{14}$

Initial treatment involves high-dose IV penicillin and metronidazole, or IV clindamycin monotherapy, with conversion to 2-6 weeks of oral therapy after the patient's clinical condition has stabilized. ${ }^{8}$ Heparin is given only in cases where retrograde propagation of thrombosis to the cavernous sinus has occurred, since heparin may actually disseminate the infection. ${ }^{15}$ Standard supportive care for respiratory and hemodynamic compromise is important.

Although almost universally fatal in Lemierre's era, ${ }^{1}$ earlier detection through diagnostic imaging, aggressive use of intravenous antibiotics and improved critical care modalities have reduced the mortality of this syndrome to approximately $8 \% .^{16}$ There is some suggestion ${ }^{16-19}$ that the incidence of Lemierre syndrome is increasing since practitioners are becoming less liberal in their use of antibiotics for upper respiratory infections. If this is true, prudent emergency physicians should be increasingly alert for signs of jugular thrombosis and metastatic septic disease following acute otolaryngologic infections.

\section{Conclusion}

Lemierre syndrome is an uncommon but potentially lethal complication of otolaryngological infections. Early recognition and aggressive antibiotic therapy are critical elements in reducing mortality.

Competing interests: None declared.

\section{References}

1. Lemierre A. On certain septicemias due to anaerobic organisms. Lancet 1936:701-3.

2. Sinave CP, Hardy GJ, Fardy PW. The Lemierre syndrome: suppurative thrombophlebitis of the internal jugular vein secondary to oropharyngeal infection. Medicine 1989;68:85-94.
3. Vandenberg SJ, Hartig GK. Lemierre's syndrome. Otolaryngol Head Neck Surg 1998;119(5):516-8.

4. Gwaltney JM Jr, Bisno AL. Pharyngitis. In: Mandell GL, Bennett JE, Dolin R, editors. Principles and practice of infectious diseases, 5th ed. Philadelphia: Churchill Livingston; 2000. p. 658.

5. Tan NC, Tan DY, Tan LC. An unusual headache: Lemierre's Syndrome. J Neurol 2003;250:245-6.

6. Maalikjy Akkawi N, Borroni B, Magoni M, Vignolo LA, Padovani A. Lemierre's syndrome complicated by carotid thrombosis. Neurol Sci 2001;22:403-4.

7. McLean AS, Tyler K. Cardiac tamponade in a postpartum woman with Lemierre's Syndrome. Anaesth Intensive Care 1998;26:582-3.

8. Hagelskjaer Kristnesen L, Prag J. Human necrobacillosis, with emphasis on Lemierre's syndrome. Clin Infect Dis 2000;31:524-32.

9. Hagelskjaer LH, Prag J, Malczynski J, Kristensen JH. Incidence and clinical epidemiology in necrobacillosis, including Lemierre's syndrome, in Denmark 1990-1995. Eur J Clin Microbiol Infect Dis 1998;17:561-5.

10. Gudinchet F, Maeder P, Neveceral P, Schnyder P. Lemierre's Syndrome in children: high-resolution CT and color Doppler sonography patterns. Chest 1997;112:271-3.

11. Fiesseler FW. Pharyngitis followed by hypoxia and sepsis: Lemierre syndrome. Am J Emerg Med 2001;19:320-2.

12. De Lima JE Jr, Levin M. Lemierre's Syndrome: post-anginal septicemia. Ped Radiol 2003;33:281-3.

13. Albertyn LE, Alcock MK. Diagnosis of internal jugular vein thrombosis. Radiology 1987;162:505-8.

14. Lustig LR, Cusick BC, Cheung SW, Lee KC. Lemierre's syndrome: two cases of postanginal sepsis. Otolaryngol Head Neck Surg 1995;112;767-72.

15. Lee BK, Lopez F, Genovese M, Loutit JS. Lemierre's Syndrome. South Med J 1997;90(6):640-3.

16. Smith SA. Respiratory failure as a complication of pharyngitis: Lemierre's Syndrome. Ped Emerg Care 1999;15(6):402-3.

17. Moore BA, Deckle C, Werkhaven J. Bilateral Lemierre's syndrome: a case report and literature review. Ear Nose Throat J 2002;81:234-6,238-40,242.

18. Jane R. Critical care nurses be aware: Lemierre's syndrome is on the rise. Aust Crit Care 2003;16:126-32.

Correspondence to: Dr. Jonnathan M. Busko, 4525 Statesville Rd., Charlotte NC 28269; 704 943-6065, fax 704 963-6001, jonnathanb@medic911.com 